\title{
The South African Vascular Surgical Cardiac Risk Index (SAVS-CRI): A prospective observational study
}

\author{
Y Moodley, ${ }^{1}$ MMedSci; P Naidoo, ${ }^{2}$ MB ChB, FCP (SA); B M Biccard, ${ }^{1}$ MB ChB, FCA (SA), FFARCSI, MMedSci, PhD \\ ${ }^{1}$ Perioperative Research Group, Department of Anaesthetics, Nelson R Mandela School of Medicine, University of KwaZulu-Natal and \\ Inkosi Albert Luthuli Central Hospital, Durban, South Africa \\ Department of Chemical Pathology, National Health Laboratory Services, Inkosi Albert Luthuli Central Hospital, Durban, South Africa
}

Corresponding author: Y Moodley(moodleyyo@ukzn.ac.za)

\begin{abstract}
Background. Recent evidence suggests that application of the Revised Cardiac Risk Index (RCRI) for peri-operative cardiovascular risk stratification in vascular surgery patients may be inappropriate, necessitating the development of risk indices specific to vascular surgery patients. objectives. To identify risk factors for cardiovascular morbidity and mortality in South African patients undergoing major vascular surgery, and to develop an appropriate cardiovascular risk stratification index, the South African Vascular Surgical Cardiac Risk Index (SAVS-CRI), which could be used to predict the risk of peri-operative major adverse cardiovascular events (MACEs) in South African vascular surgery patients.

Methods. We prospectively collected data related to peri-operative MACE occurrence and established risk factors for peri-operative MACEs from adult patients who underwent elective vascular surgery at a tertiary hospital in Durban, South Africa, between February 2008 and March 2011. We determined independent predictors of peri-operative MACEs in our cohort by binary logistic regression and used the identified predictors to create a risk index that stratified patients into low-, intermediate- or high-risk groups.

Results. Six independent predictors of peri-operative MACEs were identified in the vascular surgery cohort: age $>65$ years, a history of ischaemic heart disease, a history of diabetes, chronic $\beta$-blockade, prior coronary revascularisation, and the vascular surgical procedure. The risk model derived from these risk factors appeared to discriminate between the three risk groups more accurately than the RCRI. Conclusion. The RCRI is not appropriate for peri-operative cardiovascular risk stratification in vascular surgery patients. The SAVS-CRI may be preferable for risk stratification in South African vascular surgery patients, although independent validation is required.
\end{abstract}

S Afr Med J 2013;103(10):746-750. DOI:10.7196/SAMJ.6967

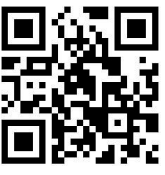

It is estimated that 234 million major non-cardiac surgical procedures are undertaken worldwide each year, ${ }^{[1]}$ with rates of cardiac morbidity or mortality following these procedures ranging between $0.5 \%$ and $30 \% \cdot{ }^{[2]}$ Pre-operative cardiac evaluation and prognostication using clinical risk scores may allow physicians to reduce cardiac risk following non-cardiac surgery. Risk stratification allows physicians to identify patients at risk for cardiac complications, optimise treatment of co-morbid conditions before surgery, or offer high-risk patients the option of conservative management rather than surgery. ${ }^{[3]}$

Lee's Revised Cardiac Risk Index (RCRI) has superseded several early risk stratification models. ${ }^{[4-6]}$ The RCRI consists of six independent predictors of major adverse cardiac events (MACEs) following non-cardiac surgery: ischaemic heart disease, cerebrovascular accident, congestive heart failure, renal impairment, major surgery and diabetes. The peri-operative risk of MACEs depends on the number of risk factors present, ranging between approximately $0.5 \%$ (no risk factors present) and $11 \%$ (three or more risk factors present). Although it is widely utilised and has been validated in several surgical populations, the appropriateness of the RCRI for risk stratification in some populations remains debatable. A recent meta-analysis by Ford et al. ${ }^{[7]}$ found that although the RCRI was able to discriminate well between low- and high-risk patients who underwent mixed non-cardiac surgery, it performed poorly in patients who underwent vascular surgery. The Vascular Study Group of New England (VSG) developed a risk scoring model, the VSG cardiac risk index (VSG-CRI), for vascular surgical patients from a retrospective cohort which appears to perform better than the RCRI at predicting peri-operative MACEs in vascular surgery patients. ${ }^{[8]}$
This clinical scoring system identified nine clinical variables, namely age, smoking, prior surgical intervention for coronary artery disease, diabetes, ischaemic heart disease, heart failure, chronic $\beta$-blockade, chronic obstructive airway disease and renal impairment, to stratify vascular surgery patients into increasing levels of cardiac risk. However, the VSG-CRI requires prospective validation prior to its use in clinical practice.

While the RCRI has been validated in European and American surgical populations, data from developing countries remain scant. In addition, shifts in disease patterns are becoming more common in developing countries. This epidemiological transition may impact on the importance and weighting of several risk factors associated with MACEs following non-cardiac surgery between developing and developed countries. ${ }^{\left[{ }^{[9]}\right.}$ A retrospective study by Biccard and Bandu ${ }^{[9]}$ found that of all the RCRI risk factors, only elevated serum creatinine was a predictor of cardiac mortality in South African (SA) vascular surgery patients. A history of smoking, which is not an RCRI risk factor, was also found to be a predictor of cardiac mortality in the same study. These conflicting findings necessitate further research regarding risk factors for peri-operative MACEs in SA vascular surgical patients.

\section{Objectives}

- Primary: To determine clinical risk predictors of MACEs following vascular surgery in a SA cohort through an adequately powered, prospective, observational study design.

- Secondary: To develop an appropriate cardiovascular risk stratification index that could be used to predict the risk of perioperative MACEs in SA vascular surgery patients. 


\section{Methods}

\section{Setting, patients and outcomes}

Ethical approval for this study was obtained from the Biomedical Research Ethics Committee of the University of KwaZulu-Natal, Durban, SA. The study was conducted at the Inkosi Albert Luthuli Central Hospital (IALCH), Durban. The hospital provides a tertiary/quaternary service to patients living in the province of KwaZulu-Natal. A registry of consenting adult patients who underwent elective vascular surgery at IALCH between February 2008 and March 2011 was created to prospectively collect data related to demographic variables, established cardiac risk factors for peri-operative MACEs, ${ }^{[4,8}$ and other possible clinical risk factors such as hypertension. ${ }^{[3]}$ Definitions of established cardiac risk factors for perioperative MACEs were adopted from the study of Lee et al. ${ }^{[4]}$

Patients' troponin-I levels were measured on the first 3 postoperative days, with a level of $\geq 0.1 \mathrm{ng} / \mathrm{ml}$ considered a positive result. ${ }^{[10]}$ The primary outcome was a MACE, defined as a composite of death within 30 days of surgery or peri-operative troponin-I leak. We have used this definition of MACE in a previous publication that used this dataset. ${ }^{[10]}$

\section{Statistical analysis}

Independent Student's $t$-tests and analysis of variance (ANOVA) were used to analyse continuous data, expressed as means ( \pm standard deviation $(\mathrm{SD})$ ) or medians (interquartile range (IQR)). Categorical data were analysed using descriptive statistics and $\chi^{2}$ or Fisher's exact tests, where appropriate. Friedman's two-way ANOVA was used to describe the postoperative troponin-I leaks. Clinical risk factors for peri-operative MACEs were determined by binary logistic regression, which included all the potential clinical risk predictors identified in the RCRI ${ }^{[4]}$ and the VSG-CRI ${ }^{[8]}$ models (with the exception of data related to chronic obstructive pulmonary disease, which were not collected), and previous SA retrospective studies. ${ }^{[3,9]}$ There were $>10$ outcome events per variable, which enabled us to enter all potential clinical predictors into the regression equation, ${ }^{[11]}$ along with a surgical 'procedural severity' risk factor. Surgical procedures were classified as carotid interventions (reference surgery group), extraperitoneal vascular procedures (intermediate-risk surgery group) and intraperitoneal vascular surgery (high-risk surgery group). Results are presented as odds ratios (ORs) with 95\% confidence intervals (CIs).
A clinical score based on the final logistic regression model was developed using the method described by Sullivan et al. ${ }^{[12]}$ Age was converted into a dichotomous variable for the clinical risk model, using the optimal discriminatory point identified on a receiver operating characteristic (ROC) curve. The weighted point score for each clinical variable was calculated by dividing each $\beta$-coefficient by the smallest $\beta$-coefficient in the analysis. The predictive accuracy of our model was evaluated by ROC curve analysis. ${ }^{[13]}$ The diagnostic and general optimal test cutoffs were used to determine three risk categories (low, intermediate and high risk). The general optimal test cut-off value is the point where the rate of true positives is optimised while minimising the rate of false positives, thereby reflecting the point with the highest accuracy for prediction of the study's primary outcome. This was defined by ROC statistics using a 1:1 weighting of sensitivity and specificity and the point

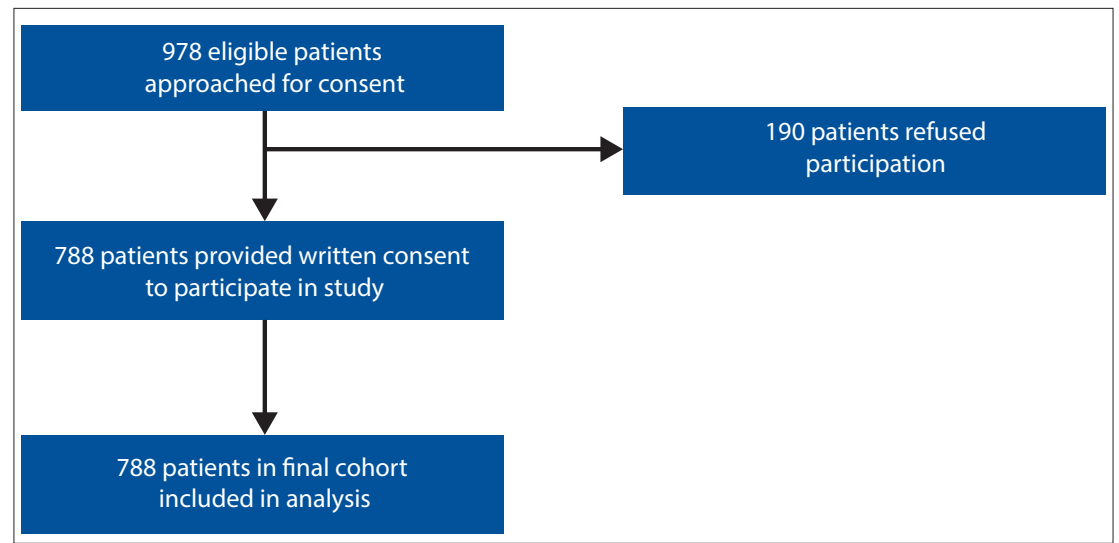

Fig. 1. Flow diagram of recruited patients.

Table 1. Baseline patient clinical characteristics

\begin{tabular}{|c|c|c|c|c|}
\hline \multirow[b]{2}{*}{ Patient characteristic } & \multirow[b]{2}{*}{ Total cohort $(N=788)$} & \multicolumn{2}{|c|}{ Patients with/without peri-operative MACE } & \multirow[b]{2}{*}{$p$-value } \\
\hline & & With $(N=136)$ & Without $(N=652)$ & \\
\hline Males, $n(\%)$ & $512(65)$ & $80(58.8)$ & $432(66.3)$ & 0.114 \\
\hline Age (years), mean $( \pm S D)$ & $58.3( \pm 14.2)$ & $62.4( \pm 13.4)$ & $57.4( \pm 14.3)$ & 0.001 \\
\hline History of ischaemic heart disease, $n(\%)$ & $275(34.9)$ & $74(54.4)$ & $201(30.8)$ & $<0.001$ \\
\hline Diabetes, $n(\%)$ & $338(42.9)$ & $78(57.4)$ & $260(39.9)$ & $<0.001$ \\
\hline History of congestive heart failure, $n(\%)$ & $37(4.7)$ & $11(8.1)$ & $26(4)$ & 0.046 \\
\hline History of CVA, $n(\%)$ & $159(20.2)$ & $23(16.9)$ & $136(20.9)$ & 0.348 \\
\hline Renal impairment ( $N=730), n(\%)$ & $18(2.5)$ & $4(3.1)$ & $14(2.3)$ & 0.543 \\
\hline Hypertension, $n(\%)$ & $540(68.5)$ & $107(78.7)$ & $433(66.4)$ & 0.005 \\
\hline History of smoking, $n(\%)$ & $546(69.3)$ & $86(63.2)$ & $460(70.6)$ & 0.102 \\
\hline Chronic $\beta$-blockade, $n(\%)$ & $267(33.9)$ & $82(60.3)$ & $185(28.4)$ & $<0.001$ \\
\hline History of prior coronary revascularisation, $n(\%)^{*}$ & $47(6)$ & $9(6.6)$ & $38(5.8)$ & 0.692 \\
\hline
\end{tabular}


determined by the value with the minimum distance when using the formula: Distance $=(1-\text { sensitivity })^{2}+(1-\text { specificity })^{2} \cdot{ }^{[14]}$ The diagnostic cut-off point was chosen at a specificity of $95 \%$ while optimising sensitivity. ${ }^{[14]}$

All statistical analyses were performed using SPSS version 19.

\section{Results}

Of the 978 patients eligible for the study, 788 consented and were included in the final risk model cohort (Fig. 1). There was a peri-operative MACE rate of $17.3 \%$ (136/788 patients). Of the 136 patients with peri-operative MACEs, 43 (31.6\%) died within 30 days of their surgery.

The medical characteristics of the patients are shown in Table 1. More than two-thirds were male. Increasing age, and a history of ischaemic heart disease, diabetes, hypertension, congestive heart failure and $\beta$-blocker prescription, had univariate associations with peri-operative MACEs.

The characteristics of the troponin-I leak in 136 patients with perioperative MACEs are shown in Table 2. There were no significant differences in postoperative troponin-I measurements between days $(p=0.64)$.

Six independent risk factors for peri-operative MACEs were identified: age, a history of ischaemic heart disease, chronic $\beta$-blockade, a history of diabetes, a prior history of coronary revascularisation, and the vascular surgical procedure (Table 3). The SAVS-CRI points scoring system is also shown in Table 3.

Table 2. Postoperative troponin-I measurements at days 1,2 and 3

\begin{tabular}{lll}
\hline & \multicolumn{2}{c}{ Troponin-I $(\mathrm{ng} / \mathrm{ml})$} \\
\cline { 2 - 3 } Postoperative day & Median & Interquartile range \\
\hline Day 1 & 0.33 & $0.04-2.15$ \\
Day 2 & 0.82 & $0.17-4.30$ \\
Day 3 & 0.84 & $0.17-3.42$
\end{tabular}

The proportion of peri-operative MACEs associated with the low-, intermediate- and high-risk RCRI categories as proposed by the American College of Cardiology/American Heart Association (ACC/ AHA) and the SAVS-CRI categories are shown in Tables 4 and 5, respectively. There were significant differences in the proportions of peri-operative MACEs between categories in each index $(p<0.001$ for the RCRI categories and $p<0.001$ for the SAVS-CRI categories). The area under the curve (AUC) for the RCRI categories was 0.59 (95\% CI $0.54-0.64 ; p=0.001)$ and that for the SAVS-CRI was 0.736 (95\% CI $0.69-0.78 ; p<0.001$ )

The results of the multivariate analysis for the SAVS-CRI model and the RCRI ${ }^{[4]}$ categories are shown in Table 6. Our index was able to predict peri-operative MACEs more accurately in all three risk categories than Lee's RCRI. ${ }^{[4]}$

\section{Discussion}

The main finding of this study was that the SAVS-CRI appears to perform better than the RCRI in risk-stratifying SA vascular surgical patients. The AUC of the SAVS-CRI does not overlap with that of the RCRI, and the CIs of the SAVS-CRI are discrete in comparison with the RCRI risk categories in this cohort.

The clinical risk factors for peri-operative MACEs in SA vascular surgery patients differ in their importance from the clinical risk factors described in the international literature. Our data suggest that of the $\mathrm{RCRI}^{[4]}$ risk factors, only a history of diabetes and ischaemic heart disease are independently associated with peri-operative MACEs. Other risk factors, such as smoking, are unlikely to be robust risk predictors because they do not characterise the end-organ damage associated with cardiovascular disease in the same way that established risk factors (such as a history of ischaemic heart disease) do. ${ }^{[15]}$ Adoption of the VSG-CRI for SA patients is also inappropriate, as only 5 of the 9 clinical variables identified in the VSG-CRI study ${ }^{[8]}$ were independently associated with peri-operative MACEs in our study.

Our data also suggest that the weighting of the risk factors associated with cardiac morbidity differs in SAs when compared with the VSG-CRI ${ }^{[8]}$ which is consistent with previous suggestions that the

Table 3. Independent risk factors for peri-operative MACEs following vascular surgery

\begin{tabular}{llll}
\hline Patient characteristic & OR $(\mathbf{9 5} \% \mathbf{C I})$ & $p$-value & SAVS-CRI point score \\
\hline Clinical risk factors in the model & & & \\
Age $(>65$ years $)$ & $2.014(1.3-3.121)$ & 0.002 & 2 \\
History of ischaemic heart disease & $1.875(1.178-2.986)$ & 0.008 & 2 \\
Chronic $\beta$-blockade & $3.237(2.056-5.095)$ & $<0.001$ & 4 \\
Diabetes & $1.830(1.125-2.979)$ & 0.015 & 2 \\
Prior surgical intervention for coronary artery disease & $0.406(0.165-1)$ & 0.050 & -3 \\
Intermediate-risk surgery & $2.556(1.131-5.775)$ & 0.024 & 3 \\
Open supra-inguinal vascular surgery & $8.354(3.105-22.475)$ & $<0.001$ & 7 \\
Clinical risk factors not in the model & & & - \\
Male gender & $0.889(0.529-1.495)$ & 0.657 & - \\
Smoker & $0.857(0.499-1.471)$ & 0.576 & - \\
Congestive cardiac failure & $1.358(0.593-3.112)$ & 0.470 & - \\
Cerebrovascular accident & $1.037(0.541-1.990)$ & 0.912 & - \\
Hypertension & $0.740(0.416-1.315)$ & 0.305 & - \\
Creatinine $>177 \mu$ mol/ 1 & $1.438(0.396-5.222)$ & 0.581 & \\
OR = odds ratio; CI = confidence interval; SAVS-CRI = South African Vascular Surgical Cardiac Risk Index; MACE = major adverse cardiovascular event. &
\end{tabular}


Table 4. RCRI risk categories and associated proportions of MACEs following vascular surgery

\begin{tabular}{lll}
\hline Risk group & Score range & $\begin{array}{l}\text { Proportion of MACEs within } \\
\text { risk group (95\% CI) }\end{array}$ \\
\hline Low & 0 & $0.11(0.07-0.15)$ \\
Intermediate & $1-2$ & $0.20(0.16-0.24)$ \\
High & $>2$ & $0.28(0.17-0.39)$ \\
$\begin{array}{l}\text { RCRI = Revised Cardiac Risk Index; MACE = major adverse cardiovascular event; } \\
\text { CI = confidence interval. }\end{array}$
\end{tabular}

Table 5. SAVS-CRI risk categories and associated proportions of MACEs following vascular surgery

\begin{tabular}{lll}
\hline Risk group & Score range & $\begin{array}{l}\text { Proportion of MACEs within } \\
\text { risk group }(95 \% \text { CI })\end{array}$ \\
\hline Low & $<7$ & $0.07(0.04-0.09)$ \\
Intermediate & $7-11$ & $0.21(0.16-0.26)$ \\
High & $>11$ & $0.47(0.38-0.56)$ \\
$\begin{array}{l}\text { SAVS-CRI = South African Vascular Surgical Cardiac Risk Index; MACE = major adverse } \\
\text { cardiovascular event; CI = confidence interval. }\end{array}$
\end{tabular}

\section{Table 6. Comparison of models (RCRI v. SAVS-CRI)}

\begin{tabular}{llll}
\hline Model & Risk group & OR $(95 \% \mathrm{CI})$ & $p$-value \\
\hline RCRI & Low & 1 (reference) & 0.002 \\
& Intermediate & $1.999(1.268-3.152)$ & 0.003 \\
& High & $2.998(1.539-5.839)$ & 0.001 \\
\multirow{2}{*}{ SAVS-CRI } & Low & 1 (reference) & $<0.001$ \\
& Intermediate & $3.541(2.179-5.753)$ & $<0.001$ \\
& High & $11.771(6.980-19.850)$ & $<0.001$
\end{tabular}

RCRI $=$ Revised Cardiac Risk Index; SAVS-CRI = South African Vascular Surgical Cardiac Risk Index; $\mathrm{OR}=$ odds ratio; $\mathrm{CI}=$ confidence interval.

SA risk profile may differ from that of North American and European populations. ${ }^{[3]}$ This suggests that clinical indices of peri-operative cardiovascular risk that are not derived in SA may have inferior performance when applied to SAs. ${ }^{[3]}$

When compared with the cohorts of Lee et al. ${ }^{[4]}$ and Boersma et al. ${ }^{[16]}$ the prevalence of cardiac risk predictors in our surgical cohort was similar or higher, with the exception of congestive heart failure. Since the study of Lee et al. ${ }^{[4]}$ congestive heart failure has been considered a significant risk factor for peri-operative cardiovascular events in patients undergoing non-cardiac surgery. It is possible that congestive cardiac failure may be an important risk factor; however, we could not demonstrate this in our study. This may be due to the small sample size, a potential bias to manage some of these patients conservatively, and the notoriously difficult clinical diagnostic criteria. ${ }^{[15]}$

Multivariable analysis of our study data suggests that age, a history of ischaemic heart disease, diabetes, chronic $\beta$-blockade, prior coronary revascularisation and the vascular surgical procedure are important independent clinical risk predictors of cardiac morbidity/mortality in SA vascular surgery patients. Belmont et al ${ }^{[17]}$ also found age to be associated with 30-day mortality and complications following lower limb amputation (OR 1.03, 95\% CI $1.02-1.05$; $p<0.0001$ ). McFalls et al. ${ }^{[18]}$ showed that age $>70$ years was an independent predictor of troponin leak during the peri-operative period in a vascular surgery cohort with documented coronary artery disease (OR 1.84, 95\% CI $1.14-2.98 ; p=0.01$ ). Increasing age is associated with an increased prevalence of co-morbidities such as hypertension, heart disease and stroke. When Lee's RCRI was adapted to include age, the adapted index showed improved cardiovascular mortality prediction. ${ }^{[19]}$ In addition to the abovementioned studies, our study confirms the significance of an increased duration of exposure to cardiovascular risk factors as a function of age on peri-operative cardiovascular morbidity/mortality in vascular surgery patients. ${ }^{[15]}$

Ischaemic heart disease and diabetes have long been identified as independent predictors of peri-operative MACEs in several previous studies of vascular surgery cohorts. ${ }^{[4,1,1,1]}$ Coronary artery stenosis may aggravate myocardial ischaemia in the peri-operative period. Furthermore, in patients with coronary artery disease there may be peri-operative atherosclerotic plaque rupture and resulting thromboembolism-associated myocardial infarction. ${ }^{[20]}$

Although $\beta$-blockade has been shown to be beneficial in reducing peri-operative myocardial ischaemia and arrhythmias, ${ }^{[2,22]}$ there is some evidence to suggest that chronic $\beta$-blocker use may have detrimental effects after major surgery. A meta-analysis by Giles et al. ${ }^{[23]}$ found a significant association between chronic $\beta$-blockade and peri-operative myocardial infarction (OR 2.14, 95\% CI 1.29 - 3.56). In agreement with this meta-analysis, the VSG-CRI study, ${ }^{[8]}$ studies by Biccard and Pooran ${ }^{[24]}$ and Hoeks et al., ${ }^{[25]}$ and our study have also found chronic $\beta$-blockade to be associated with peri-operative MACEs. There may be several explanations for our observations. Chronic $\beta$-blockade is associated with an up-regulation of $\beta$-adrenoreceptors. ${ }^{[26]}$ As such, patients receiving a chronic $\beta$-blocker may be more likely to develop a peri-operative tachycardia than their acutely $\beta$-blocked peers. Peri-operative tachycardia may result in peri-operative myocardial ischaemia at lower than expected heart rates. ${ }^{[23]}$ In addition, patients may not be able to take their chronic oral medications, such as $\beta$-blockers, after their operations. ${ }^{[27]}$ This would equate to withdrawal of chronic $\beta$-blockade, which in the peri-operative period is associated with an increased likelihood of short-term MACEs and mortality, ${ }^{[28]}$ as well as increased mortality at 1 year post-surgery ${ }^{[25]}$ Our observed association of chronic $\beta$-blockade with peri-operative MACEs may therefore be due to the impact of $\beta$-blocker withdrawal in the peri-operative period. When compared with the VSG-CRI study, ${ }^{[8]}$ chronic $\beta$-blockade appears to be associated with more risk in our patients, perhaps reflecting more frequent withdrawal of chronic $\beta$-blockade.

The benefit of prophylactic pre-operative coronary revascularisation prior to surgery remains debatable. Two prospective studies (the Coronary Artery Revascularization Prophylaxis and Dutch Echocardiographic Cardiac Risk Evaluation Applying Stress Echo-V studies ${ }^{[2,300]}$ have shown that prophylactic coronary revascularisation before major vascular surgery does not reduce the risk of peri-operative myocardial infarction. However, in vascular patients with an incidental history of prior coronary revascularisation, the VSG-CRI study group found it to be associated with a protective effect against peri-operative MACEs. ${ }^{[8]}$ Our findings are in agreement with the VSG-CRI study and suggest that coronary revascularisation, if indicated prior to major vascular surgery, may reduce peri-operative cardiovascular risk. As coronary artery disease contributes proportionally more to risk in our patients, it is not surprising that prior coronary revascularisation appears to provide greater protection in our patients than was seen in the VSG-CRI study. ${ }^{[8]}$

The SAVS-CRI appears to have superior clinical performance compared with the RCRI in SA vascular surgical patients, as evidenced by the significantly higher AUC in the ROC curve analysis and a significantly different incidence of MACEs between risk categories.

The observed peri-operative MACE rates may be considered high across all three risk categories. This may partly be due to our definition of MACE, which only required a troponin leak 
above the upper reference limit. Troponin-I is a highly sensitive biomarker of myocardial damage, and concerns have been raised regarding overestimation of the extent of perceived myocardial damage following an abnormal but low troponin-I measurement. However, even a perceived minor troponin leak of this nature has prognostic importance, as recently confirmed in the Vascular events In noncardiac Surgery patIents cOhort evaluatioN (VISION) Study. ${ }^{[3]]}$ Previously, Le Manach et al. observed that patients with abnormal but low postoperative troponin-I levels are at increased risk of a 'delayed' peri-operative myocardial infarction and mortality ${ }^{[32]}$ Duration of hospital stay and mortality were also significantly increased in the group with a diagnosis of myocardial damage (a troponin leak that did not fulfil the study criteria for myocardial infarction) compared with patients without a troponin leak. ${ }^{[3]]}$ The results of the VISION study showed that even peak troponin values far lower than the commonly used threshold for a 'medical' myocardial infarction following non-cardiac surgery are a strong independent predictor of 30 -day mortality, with over $40 \%$ of deaths being attributable to elevated postoperative troponins. ${ }^{[3]}$

Furthermore, approximately half of the patients who died following a troponin leak during the VISION study died of non-vascular causes. Myocardial injury may decrease the likelihood of surviving subsequent non-vascular peri-operative complications, such as pneumonia, and could explain the association observed between postoperative troponin leak and all-cause mortality in the VISION study. ${ }^{[3]}$ This is supported by the observation that the complications resulting in the majority of noncardiovascular deaths have often developed later in the clinical course, following an earlier troponin leak. ${ }^{[3]]}$ For these reasons, we believe the definition of MACE used in this study to be clinically relevant.

The results of this study require prospective validation in an independent cohort.

\section{Study strengths}

We prospectively collected data on potential cardiac risk factors over a 3-year period in SA vascular surgical patients. The dataset was complete for all patients who consented to participate in the study.

\section{Study limitations}

Data on chronic obstructive pulmonary disease, which was an independent predictor of adverse outcomes in the VSG-CRI model, were not collected. We were also unable to assess postoperative compliance with chronic medication and hence can only speculate on the withdrawal of $\beta$-blockers and any association with peri-operative MACEs. The impact of HIV-associated vasculopathy on peri-operative MACEs could not be investigated owing to the small sample $(n=73)$ of known HIV-positive patients. Another limitation is that our study was an analysis of data from a single clinical trial site; larger studies, comprising multiple clinical trial sites in different geographical areas in SA, are required to validate the general application of the SAVS-CRI.

\section{Conclusion}

The SAVS-CRI identified six independent predictors of MACEs. Importantly, in agreement with the VSG-CRI model, only two of these risk factors were identified in the RCRI, while the other three risk factors were also identified in the VSG-CRI. This study confirms that it is probably inappropriate to risk-stratify SA vascular surgical patients using the RCRI. Development and validation of a suitable cardiac risk index for use in SA patients undergoing vascular surgery is therefore necessary.

Acknowledgement. The study was funded by a Medical Research Council self-initiated research grant awarded to Bruce M Biccard.

\section{References}

1. Weiser TG, Regenbogen SE, Thompson KD, et al. An estimation of the global volume of surgery: A modelling strategy based on available data. Lancet 2008;372(9633):139-144. [http://dx.doi. org/10.1016/s0140-6736(08)60878-8]

2. Bakker EJ, Ravensbergen NJ, Poldermans D. Perioperative cardiac evaluation, monitoring, and risk reduction strategies in noncardiac surgery patients. Curr Opin Crit Care 2011;17(5):409-415. [http:// dx.doi.org/10.1097/MCC.0b013e328348d40f]

3. Biccard BM, Nepaul S. Risk factors associated with intermediate and long-term mortality following vascular surgery in South African patients. Cardiovasc J Afr 2010;21(5):263-267. [http://dx.doi.org/Cvj-21.004]

4. Lee TH, Marcantonio ER, Mangione CM, et al. Derivation and prospective validation of a simple Lee TH, Marcantonio ER, Mangione CM, et al. Derivation and prospective validation of a simple
index for prediction of cardiac risk of major noncardiac surgery. Circulation 1999;100(10):1043-1049. index for prediction of cardiac risk of major non
[http://dx.doi.org/10.1161/01.CIR.100.10.1043]

5. Fleisher LA, Beckman JA, Brown KA, et al. ACC/AHA 2007 guidelines on perioperative cardiovascular . Fleisher LA, Beckman JA, Brown KA, et al. ACC/AHA 2007 guidelines on perioperative cardiovascular
evaluation and care for noncardiac surgery: A report of the American College of Cardiology/American evaluation and care for noncardiac surgery: A report of the American College of Cardiology/American
Heart Association Task Force on Practice Guidelines (Writing Committee to Revise the 2002 Guidelines on Perioperative Cardiovascular Evaluation for Noncardiac Surgery) developed in collaboration with the American Society of Echocardiography, American Society of Nuclear Cardiology, Heart Rhythm Society, Society of Cardiovascular Anesthesiologists, Society for Cardiovascular Angiography an Interventions, Society for Vascular Medicine and Biology, and Society for Vascular Surgery. J Am Coll Cardiol 2007;50(17):e159-241. [http://dx.doi.org/10.1016/j.jacc.2007.09.003]

6. Poldermans D, Bax JJ, Boersma E, et al. Guidelines for pre-operative cardiac risk assessment and perioperative cardiac management in non-cardiac surgery. Eur Heart J 2009;30(22):2769-2812. [http:// dx.doi.org/10.1093/eurheartj/ehp337]

7. Ford MK, Beattie WS, Wijeysundera DN. Systematic review: Prediction of perioperative cardiac complications and mortality by the revised cardiac risk index. Ann Intern Med 2010;152(1):26-35. complications and mortality by the revised cardiac risk index.
[http://dx.doi.org/10.1059/0003-4819-152-1-201001050-00007]

8. Bertges DJ, Goodney PP, Zhao Y, et al. The Vascular Study Group of New England Cardiac Risk Index (VSGBertges DJ, Goodney PP, Zhao Y, et al. The Vascular Study Group of New England Cardiac Risk Index (VSG-
CRI) predicts cardiac complications more accurately than the Revised Cardiac Risk Index in vascular surgery CRI) predicts cardiac complications more accurately than the Revised Cardiac Risk Index in vascular surg
patients. J Vasc Surg 2010;52(3):674-683, 683 e671-683 e673. [http://dx.doi.org/10.1016/j.jvs.2010.03.031]

patients. J Vasc Surg 2010;52(3):674-683, 683 e671-683 e673. [http://dx.doi.org/10.1016/.j.js.2010.03.031]
9iccard BM, Bandu R. Clinical risk predictors associated with cardiac mortality following vascular surgery in South African patients. Cardiovasc J Afr 2007;18(4):216-220.

10. Biccard BM, Naidoo P, de Vasconcellos K. What is the best pre-operative risk stratification tool for major adverse cardiac events following elective vascular surgery? A prospective observational cohor study evaluating pre-operative myocardial ischaemia monitoring and biomarker analysis. Anaesthesia 2012;67(4):389-395. [http://dx.doi.org/10.1111/j.1365-2044.2011.07020.x]

11. Peduzzi P, Concato J, Kemper E, Holford TR, Feinstein AR. A simulation study of the number of events per variable in logistic regression analysis. J Clin Epidemiol 1996;49(12):1373-1379. [http:// dx.doi.org/10.1016/s0895-4356(96)00236-3]

12. Sullivan LM, Massaro JM, D’Agostino RB, Sr. Presentation of multivariate data for clinical use: The Framingham Study risk score functions. Stat Med 2004;23(10):1631-1660. [http://dx.doi.org/10.1002/sim.1742]

13. Grunkemeier GL, Jin R. Receiver operating characteristic curve analysis of clinical risk models. Ann Grunkemeier GL, jin R. Receiver operating characteristic curve analysis of clinical ris
Thorac Surg 2001;72(2):323-326. [http://dx.doi.org/10.1016/S0003-4975(01)02870-3]

14. Peat JK, Barton B. Medical Statistics: A Guide to Data Analysis and Critical Appraisal. 1st ed. Malden, Mass.: Blackwell, 2005.

15. Biccard BM, Rodseth RN. Utility of clinical risk predictors for preoperative cardiovascular risk prediction. Br J Anaesth 2011;107(2):133-143. [http://dx.doi.org/10.1093/bja/aer194]

16. Boersma E, Kertai MD, Schouten O, et al. Perioperative cardiovascular mortality in noncardia surgery: Validation of the Lee cardiac risk index. Am J Med 2005;118(10):1134-1141. [http://dx.do org/10.1016/j.amjmed.2005.01.064]

7. Belmont PJ, Jr, Davey S, Orr JD, Ochoa LM, Bader JO, Schoenfeld AJ. Risk factors for 30-day postoperative complications and mortality after below-knee amputation: A study of 2,911 patients from the national surgical quality improvement program. J Am Coll Surg 2011;213(3):370-378. [http:// dx.doi.org/10.1016/j.jamcollsurg.2011.05.019]

18. McFalls EO, Ward HB, Moritz TE, et al. Predictors and outcomes of a perioperative myocardial infarction following elective vascular surgery in patients with documented coronary artery disease: Results of the CARP trial. Eur Heart J 2008;29(3):394-401. [http://dx.doi.org/10.1093/eurhearty/ehm620]

19. Biancari F, Salenius JP, Heikkinen M, Luther M, Ylonen K, Lepantalo M. Risk-scoring method for piancari F, Salenius JP, Heikkinen M, Luther M, Ylonen K, Lepantalo M. Risk-scoring method for
predion of 30-day postoperative outcome after infrainguinal surgical revascularization for critical prediction of 30-day postoperative outcome after infrainguinal surgical revascularization for critical
lower-limb ischemia: A Finnvasc registry study. World J Surg 2007;31(1):217-225; discussion 226-217. lower-limb ischemia: A Finnvasc registry study.
[http://dx.doi.org/10.1007/s00268-006-0242-y]

20. Landesberg G, Beattie WS, Mosseri M, Jaffe AS, Alpert JS. Perioperative myocardial infarction. Circulation 2009;119(22):2936-2944. [http://dx.doi.org/10.1161/circulationaha.108.828228]

21. Wiesbauer F, Schlager O, Domanovits H, et al. Perioperative beta-blockers for preventing surgeryrelated mortality and morbidity: A systematic review and meta-analysis. Anesth Analg 2007;104(1):27 41. [http://dx.doi.org/10.1213/01.ane.0000247805.00342.21]

22. Bangalore S, Wetterslev J, Pranesh S, Sawhney S, Gluud C, Messerli FH. Perioperative beta blockers in patients having non-cardiac surgery: A meta-analysis. Lancet 2008;372(9654):1962-1976. [http:// dx.doi.org/10.1016/s0140-6736(08)61560-3]

23. Giles JW, Sear JW, Foex P. Effect of chronic beta-blockade on peri-operative outcome in patients undergoing non-cardiac surgery: An analysis of observational and case control studies. Anaesthesi 2004;59(6):574-583. [http://dx.doi.org/10.1111/j.1365-2044.2004.03706.x]

24. Biccard BM, Pooran RR. Validation of a model to predict all-cause in-hospital mortality in vascular surgical patients. Cardiovasc J Afr 2008;19(6):303-308.

25. Hoeks $\mathrm{SE}$, Scholte Op Reimer WJ, van Urk H, et al. Increase of 1-year mortality after perioperative Hoeks SE, Scholte Op Reimer WJ, van Urk H, et al. Increase of 1 -year mortality after perioperative
beta-blocker withdrawal in endovascular and vascular surgery patients. Eur J Vasc Endovasc Surg 2007;33(1):13-19. [http://dx.doi.org/10.1016/j.ejvs.2006.06.019]

2007;33(1):13-19. [http://dx.doi.org/10.1016/j.ejvs.2006.06.019]
Lopez-Sendon J, Swedberg K, McMurray J, et al. Expert consensus document on beta-adrenergic Lopez-Sendon J, Swedberg K, McMurray J, et al. Expert consensus document on beta-adrenergic
receptor blockers. Eur Heart J 2004;25(15):1341-1362. [http://dx.doi.org/10.1016/j.ehj.2004.06.002] 27. Schouten O, Hoeks SE, Welten GM, et al. Effect of statin withdrawal on frequency of cardiac events after vascular surgery. Am J Cardiol 2007;100(2):316-320. [http://dx.doi.org/10.1016/j.amjcard.2007.02.093]

28. Shammash JB, Trost JC, Gold JM, Berlin JA, Golden MA, Kimmel SE. Perioperative beta-blocke withdrawal and mortality in vascular surgical patients. Am Heart J 2001;141(1):148-153. [http:// dx.doi.org/10.1067/mhj.2001.111547]

29. McFalls EO, Ward HB, Moritz TE, et al. Coronary-artery revascularization before elective majo vascular surgery. N Engl J Med 2004;351(27):2795-2804. [http://dx.doi.org/10.1056/NEJMoa041905]

30. Poldermans $\mathrm{D}$, Schouten $\mathrm{O}$, Vidakovic $\mathrm{R}$, et al. A clinical randomized trial to evaluate the safety of a noninvasive approach in high-risk patients undergoing major vascular surgery: The DECREASE-V noninvasive approach in high-risk patients undergoing major vascular surgery: The DECREASE-V Pilot Sy. J Am Coll Cardiol 2007,49(17):1763-1769. [hlp.//dx.doi.org/10.1016/j.jacc.2006.11.052] 31. Le Manach Y, Perel A, Coriat P, Godet G, Bertrand M, Riou B. Early and delayed myocardial
infarction after abdominal aortic surgery. Anesthesiology 2005;102(5):885-891. [http://dx.doi. infarction after abdominal aortic surge
org/10.1097/00000542-200505000-00004]

32. Devereaux PJ, Chan MT, Alonso-Coello P, et al. Association between postoperative troponin levels and 30-day mortality among patients undergoing noncardiac surgery. JAMA 2012;307(21):2295-2304. [http://dx.doi.org/10.1001/jama.2012.5502]

Accepted 9 July 2013 